\title{
MOBILE PEER-TO-PEER FREQUENCY-HOPPING COMMUNICATIONS
}

\author{
Don Torrieri \\ Army Research Laboratory \\ Adelphi, MD 20783
}

\begin{abstract}
The potential performance of mobile peer-to-peer frequencyhopping communication systems in multiple-access networks is studied. Through analysis and simulation, the effects of Rayleigh fading, shadowing, spectral splatter, the number of frequency channels, spatial diversity, and thermal noise are examined.

\section{INTRODUCTION}

Mobile peer-to-peer communications are used in mobile communication networks that possess no supporting infrastructure, fixed or mobile; each user has identical signal processing capability. Peer-to-peer communications have both commercial applications [1] and important military applications, the latter primarily because of their robustness in the presence of node losses. In peer-to-peer networks using code-division multiple-access (CDMA) communications, it is desirable to use frequency hopping, primarily because frequency hopping provides resistance to multiple-access interference while not requiring power control. In contrast, direct-sequence CDMA communications are susceptible to the near-far problem in the absence of power control [2], which is not viable for peer-to-peer communications.
\end{abstract}

This paper considers a network of independent, identical, frequency-hopping systems that have omnidirectional antennas, generate the same output power, share the same carriers and frequency channels, and are nearly stationary in location over a single symbol duration.

\section{HOPPING PARAMETERS}

The duration of a hop, denoted by $T_{h}$, is equal to the sum of the dwell time $T_{d}$ and the switching time $T_{s w}$. The dwell time is the duration of the frequency-hopping pulse during which the channel symbols are transmitted. The switching time is equal to the dead time, when no signal is present, plus the rise and fall times of a pulse. Even if the switching time is absent in the transmitted signal, it will be present in the dehopped signal in the receiver because of the imperfect synchronization of received and receivergenerated waveforms. The nonzero switching time, which may include an intentional guard time, decreases the transmitted symbol duration $T_{s}$. If $T_{s o}$ is the symbol duration in the absence of frequency hopping, then $T_{s}=T_{s o}\left(T_{d} / T_{h}\right)$. The reduction in symbol duration expands the transmitted spectrum and thereby reduces the number of frequency channels within a fixed total hopping band. Since the receiver filtering will ensure that rise and fall times of pulses have durations on the order of $T_{s}$, the practical assumption that $T_{s w}>T_{s}$ is made in the subsequent analysis. Thus, each interferer transmits in at most one frequency channel during the reception of a single symbol of the desired signal. Since the overhead cost of the nonzero switching time is reduced and equalization symbols can be accommodated, it is preferable to use slow frequency hopping with many symbols per hop, rather than fast frequency hopping, for communications over fading channels.

Even in the absence of fading, there are constraints on the values of the parameters of a frequency-hopping system. It is assumed that the receiver transfer function following the dehopping is approximately rectangular with bandwidth $B$, which is equal to the bandwidth of a frequency channel. The bandwidth is determined primarily by the percentage of the signal power that must be processed by the demodulator if the demodulated signal distortion and the intersymbol interference are to be negligible. In practice, this percentage must be at least 90 percent and is often more than 95 percent. The relation between $B$ and the symbol duration may be expressed as

$$
B=\frac{\alpha}{T_{s}}
$$

where $\alpha$ is a constant determined by the signal modulation. For example, if minimum-shift keying is used, then $\alpha=0.8$ if 90 percent of the signal power is included in a frequency channel, and $\alpha=1.2$ if 99 percent is included.

Let $M$ denote the number of carriers and frequency channels, $W$ the total bandwidth occupied by the $M$ channels, and $F_{s}$ the minimum separation between the carriers in a hopset. For full protection against stationary narrowband interference and jamming, it is desirable that $F_{s} \geq B$ so that the frequency channels are nearly spectrally disjoint.

To obtain the full advantage of error-correcting codes, it is important to interleave the code symbols in such a way that the symbol errors are independent (for hard-decision decoding) or that the symbols are degraded independently (for soft-decision decoding). In frequency-hopping systems operating over a fading channel, the realization of this independence requires certain constraints among the system parameter values. Symbol errors are independent if the fading is independent in each frequency channel and each codeword symbol is transmitted in a different frequency channel. If each of the codeword symbols is transmitted at the same location in each hop dwell time, then adjacent symbols are separated by $T_{h}$. Thus, a sufficient condition for nearly independent symbol errors is

$$
T_{h} \geq T_{\text {coh }}
$$

where $T_{c o h}$ is the coherence time of the fading channel. Another sufficient condition for nearly independent symbol errors is

$$
F_{s} \geq B_{\text {coh }}
$$


where $B_{c a h}$ is the coherence bandwidth of the fading channel. For practical mobile communication networks with hopping rates exceeding 100 hops/s, (2) is rarely satisfied. In a hopset with a uniform carrier separation, $F_{s}=W / M \geq B$. Thus, (3) implies that the number of frequency channels is constrained by

$$
M \leq \frac{W}{\max \left(B, B_{c o h}\right)}
$$

if nearly independent symbol errors are to be ensured. If (4) is not satisfied, there will be a performance loss due to the correlated symbol errors. If $B \geq B_{c o h}$, equalization will be necessary; if $B<$ $B_{c o h}$, it may not be.

Let $n$ denote the length of a block codeword or the constraint length of a convolutional code. Let $T_{d e l}$ denote the maximum tolerable processing delay. Since the delay caused by coding and ideal interleaving over $n$ hops is $(n-1) T_{h}+T_{s}$ and $n$ distinct frequencies are desired,

$$
n \leq \min \left(M, 1+\frac{T_{d e l}-T_{s}}{T_{h}}\right)
$$

is required. If (5) is not satisfied, then nonideal interleaving is necessary, and some performance degradation results.

Spectral splatter is the interference produced in frequency channels other than the one being used by a frequency-hopping pulse. It is caused by the time-limited nature of transmitted pulses. The degree to which spectral splatter may cause errors depends primarily on $F_{s}$ and the percentage of the signal power included in a frequency channel.

The adjacent splatter ratio $K$ is the ratio of the power due to spectral splatter from an adjacent channel to the corresponding power that arrives at the receiver in that channel. For example, if $B$ is the bandwidth of a frequency channel that includes 97 percent of the signal power and $F_{s} \geq B$, then no more than 1.5 percent of the power from a transmitted pulse can enter an adjacent channel on one side of the frequency channel used by the pulse; therefore, $K$ $\leq 0.015$. A given maximum value of $K$ can be reduced by an increase in $F_{s}$, but eventually the value of $M$ must be reduced if $W$ is fixed. As a result, the rate at which users hop into the same channel increases. This increase may cancel any improvement due to the reduction of the spectral splatter. The opposite procedure (reducing $F_{s}$ and $B$ so that more frequency channels become available) increases not only the spectral splatter but also signal distortion and intersymbol interference, so the amount of useful reduction is limited.

\section{PROPAGATION AND PERFORMANCE}

In this paper, the propagation path losses are modeled as the result of power-law losses, shadowing, and fading [3]. In the absence of shadowing and fading, the received signal power for communications over a fixed range $r$ has an average value called the areamean power, where the average is calculated over a specified geographic area. The power-law model assumes that the area-mean power has the form

$$
P_{a}=P_{0}\left(\frac{r}{R_{0}}\right)^{-\beta}
$$

where $P_{0}$ is the power when the range is $r=R_{0}$, and $\beta$ is the power law. In the following, it is assumed that $\beta=4$, which is a typical value for an urban area. In the absence of fading, the received $l o$ cal-mean power fluctuates about the area-mean power due to shadowing, which is the effect of path-to-path differences in terrain profiles for paths of the same distance. On the basis of extensive empirical data, the local-mean power $P_{l}$ is assumed to be lognormally distributed. Let $Y=10 \log _{10} P_{l} / P_{a}$. The lognormal model implies that the probability density function of $Y$ is

$$
f_{Y}(y)=\frac{1}{\sqrt{2 \pi} \sigma_{y}} \exp \left(-\frac{y^{2}}{2 \sigma_{y}^{2}}\right)
$$

where $\sigma_{y}$ is expressed in decibels and is assumed to be the same for all mobiles. A change of variable indicates that the density of the local-mean power $P_{l}$ is

$$
f_{0}(x)=\frac{1}{\sqrt{2 \pi} \sigma_{s} x} \exp \left[-\frac{\left(\ln x-\ln P_{a}\right)^{2}}{2 \sigma_{s}^{2}}\right], \quad x \geq 0
$$

where

$$
\sigma_{s}=\frac{\sigma_{y}}{10 \log _{10} e} .
$$

Integrating (8) and substituting (6) and (9) yields the probability distribution function of the normalized local-mean power, $P_{l} / P_{0}$,

$$
F_{1}(x)=\frac{1}{2}+\frac{1}{2} \operatorname{erf}\left\{\frac{3.07}{\sigma_{y}} \ln \left[x\left(\frac{r}{R_{0}}\right)^{\beta}\right]\right\}
$$

where erf \{\} denotes the error function and $3.07=\left(10 \log _{10} e\right) / \sqrt{2}$. The fading causes a power fluctuation about the local-mean power.

It is assumed that $L$ omnidirectional antennas are deployed to achieve spatial diversity. The antennas are separated from each other by several wavelengths, so that the fading of both the desired signal and the interfering signals at one antenna is independent of the fading at the other antennas. A few wavelengths are adequate because mobiles, in contrast to base stations, tend to receive superpositions of reflected waves arriving from many random angles. Because of practical physical constraints, spatial diversity will ordinarily be effective only if the carrier frequencies exceed $1 \mathrm{GHz}$. Polarization diversity and adaptive array processing are options both above and below $1 \mathrm{GHz}$, but these techniques are not examined in this paper.

One method of combining antenna outputs is predetection combining, which requires the estimation of the signal-tointerference-plus-noise ratio (SINR) at each antenna for maximalratio combining or selection diversity and the co-phasing of the $L$ antenna outputs for maximal-ratio or equal-gain combining. Since the relative phases and power levels of the signals at the $L$ antennas may change after every hop, it is almost always impractical to implement predetection combining. As a much more practical alternative, a receiver can combine the demodulated outputs rather 
than the dehopped signals from the $L$ antennas. This postdetection combining entails the time alignment of $L$ signals, which is much simpler to implement than cophasing. The SINR estimation can be eliminated by the use of a fixed combining rule, such as equalgain or square-law combining.

The interference plus noise at each antenna output is approximated by independent bandlimited white Gaussian noise, with equivalent power given by

$$
\sigma_{T}^{2}=\sigma_{n}^{2}+\sum_{i=1}^{N} p_{u i}
$$

where $\sigma_{n}^{2}$ is the thermal noise power, $N$ is the number of active interfering users, and $p_{u i}$ is the local-mean interference power received from user $i$. The Gaussian model is reasonable, especially for large numbers of interferers, because the interference signals are asynchronous, fade independently, and experience different Doppler shifts. The total interference power is approximately uniform (white) over the receiver passband if $B T_{s}=\alpha \approx 1$. The $L$ diversity antennas are assumed to be close enough to each other that the power-law losses and shadowing are nearly the same, and thus the local-mean power from a user is the same at each antenna. Each active interfering user may actually represent a cluster of users. In this cluster, some discipline such as carrier-sense multiple access is used to ensure that there is at most one transmitted signal at any time.

The desired signal is assumed to experience Rayleigh fading. The Rayleigh fading model is appropriate under the pessimistic assumption that the propagation paths are often obstructed, and thus, the power of the direct line-of-sight signal is small compared with the reflected signal power. Thus, assuming Rayleigh fading tends to give a lower bound on network performance, whereas assuming no fading provides an upper bound. Rayleigh fading may be negligible if mobile speeds are very low, which would occur if each mobile consisted of a person walking. Shadowing would still occur but would be slowly varying over time.

To avoid spectral spreading due to amplifier nonlinearities, it is desirable for the signal modulation to have a constant envelope. Noncoherent demodulation is nearly always a practical necessity in frequency-hopping systems [4]. Accordingly, we consider the use of differential phase-shift keying (DPSK) and minimum-shift keying (MSK) or some other form of spectrally compact continuous-phase frequency-shift keying (CPFSK). Although the classical theory indicates that DPSK provides an intrinsic performance advantage over noncoherent MSK, this advantage is illusory when frequency hopping is used, because of the large bandwidth requirement of DPSK. If the total hopping bandwidth $W$ is fixed, the number of frequency channels available for DPSK signaling is much smaller than it is for MSK signaling. This reduction in frequency channels largely offsets the intrinsic advantage of DPSK when interfering users are present. Alternatively, if the bandwidth $B$ is fixed, a DPSK signal will experience more distortion and spectral splatter than an MSK signal. Another disadvantage of DPSK is due to the usual lack of phase coherence from hop to hop, which necessitates an extra phase-reference symbol at the start of every hop dwell time and thereby causes a performance loss. Furthermore, Doppler shifts and frequency instabili- ties may impair a frequency-hopping DPSK system. Thus, DPSK does not appear to be as suitable a means of modulation as MSK or CPFSK for frequency-hopping communications.

Spectrally compact binary CPFSK signals do not have enough frequency shift to be demodulated by classical noncoherent demodulators with two matched filters. Therefore, we consider frequency demodulation of MSK using a limiter-discriminator. For postdetection diversity, the outputs of $L$ disciminators are weighted and combined. The weighting is by the square of the envelope at the input to each discriminator. When the desired signal is fading and the channel parameters remain constant for at least one symbol duration, a calculation using the results of Adachi and Parsons [5] yields the symbol error probability

$$
P_{s}=\frac{1}{2} \frac{(2 L-1) ! !}{L !}\left(\frac{1}{2}+\frac{2}{3} \alpha^{2}\right)^{L}\left(\frac{p_{d}}{\sigma_{T}^{2}}\right)^{-L}
$$

where $p_{d}$ is the local-mean power of the desired signal and $\alpha=B T_{s}$. The slowly varying nature of shadowing ensures that $P_{s}$ is almost always nearly constant over $T_{d e l}$ in practical systems. The information-bit error rate following hard-decision decoding can be calculated from $P_{s}$ with well-known equations. The theoretical loss due to using postdetection rather than predetection combining has been shown to be minor [5].

In the absence of Rayleigh fading, the power that enters a receiver is equal to the local-mean power. Therefore, assuming equal-gain predetection combining of CPFSK signals with perfect cophasing, uncorrelated shadowing of each interfering signal, and independent Gaussian interference plus noise at each diversity antenna, the SINR at the input of a CPFSK discriminator is $L p_{d} / \sigma_{T}^{2}$. Thus, the symbol error probability is [4]

$$
P_{s}=\frac{1}{2} \operatorname{erfc}\left(\sqrt{\frac{\xi L p_{d}}{\sigma_{T}^{2}}}\right)
$$

where erfc ( ) is the complementary error function and $\xi$ is a constant. For MSK with $B T_{s}=\alpha=1, \xi \approx 0.38$.

Let $d$ represent the duty factor, which is defined as the probability that an interferer will degrade the reception of a symbol. Thus, $d$ is the product of the probability $p_{1}$ that an interferer is transmitting and the probability $p_{2}$ that a significant portion of the interferer's transmitted waveform occurs during the symbol interval. The probability $p_{2}$ is upper bounded and well approximated by the probability that there is any overlap in time of the interference and the symbol interval. Since $T_{s w}>T_{s}$, it follows from elementary probability that $p_{2} \approx\left(T_{d}+T_{s}\right) / T_{h}$. Therefore,

$$
d \approx p_{1}\left(\frac{T_{d}+T_{s}}{T_{h}}\right) \text {. }
$$

For voice communications with voice-activity detection, $p_{1} \approx 0.4$ is a typical value.

Because it is assumed that an interferer may transmit in any frequency channel with equal probability, the probability that power from an interferer enters the transmission channel of the desired signal is 


$$
p_{t}=\frac{d}{M}
$$

It is assumed that $M$ is sufficiently large that we may neglect the fact that a channel at one of the ends of the total band has only one adjacent channel instead of two. Consequently, the probability that the power from an interferer enters one of the two adjacent channels of the desired signal is

$$
p_{a}=\frac{2 d}{M}
$$

The probability that the power enters neither the transmission channel nor the adjacent channels is $(1-3 d / M)$.

These equations make it apparent that the performance of a frequency-hopping system depends on the ratio $M_{1}=M / d$. This ratio is called the equivalent number of channels, because any decrease in the duty factor $d$ has the same impact as an increase in the number of frequency channels; what matters for performance is this ratio.

\section{SIMULATION RESULTS}

In the simulation, the locations of the mobiles were assumed to be uniformly distributed in a circular region surrounding a specific mobile receiver. Therefore, the radial distance of a mobile from the receiver has the probability distribution function

$$
G(r)=\frac{r^{2}}{R^{2}}, \quad 0<r \leq R,
$$

where $R$ is the radius of the circle. The distance of the desired mobile was randomly selected according to this distribution with $R=R_{0}$, where $R_{0}$ is the maximum communication range and corresponds to a received area-mean signal power equal to $P_{0}$. The distance of each interfering mobile was randomly selected according to this distribution with $R=R_{1}$. The selected distance of the desired mobile was substituted into (10) as the value of $r$, and then (10) was used to randomly select the local-mean power of the desired signal at the receiver. The probabilities given by (15) and (16) were used to determine if an interfering mobile produces power in the transmission channel or in one of the adjacent channels of the desired signal. If the power enters the transmission channel, then the power level is randomly selected according to $(10)$ with the distance of the mobile substituted. If the power enters one of the adjacent channels, then the potential local-mean power level is first randomly selected via (10) and then multiplied by $K$ to determine the net interference power $p_{u i}$ that appears in (11). The effect of $P_{0}$ and $\sigma_{n}^{2}$ is determined by the minimum area-mean signal-to-noise ratio (SNR), which occurs at the maximum range $r=R_{0}$ of the desired signal and is equal to $P_{0} / \sigma_{n}^{2}$.

Once the local-mean power levels and the noise power were calculated, the symbol error probability was calculated with (11) to (13). Each simulation experiment was repeated for 10,000 trials, with different randomly selected mobile locations in each trial. The performance measure is the spatial reliability, which is defined as the fraction of trials for which $P_{s}$ is less than a specified performance threshold $E$. The spatial reliability is essentially the probability that an outage does not occur.

Figures 1 to 5 depict the results of five simulation experiments for peer-to-peer networks. The figures plot spatial reliability as a function of $N$ for various values of $L$, assuming MSK and that $\beta=4, \sigma_{y}=8 \mathrm{~dB}, \alpha=1, K=0.015, R_{0}=1$, and $R_{1}=2$. The value of $K$ results from assuming contiguous frequency channels so that $F_{s}=B$.

Figure 1 provides a baseline with which the other figures may be compared. For this figure, the assumptions are $M_{1}=250$, minimum SNR $=20 \mathrm{~dB}, E=0.01$, and Rayleigh fading, and (12) was used. The number of equivalent frequency channels $M_{1}$ could model voice communications with $M=90$ channels and $d=0.36$; alternatively, it could model continuous data communications with $M=225$ and $d=0.9$. The figure illustrates the dramatic performance improvement provided by dual spatial diversity when Rayleigh fading occurs. Further increases in diversity yield diminishing returns. One can assess the impact of the spectral splatter in this example by setting $K=0$ and observing the change in the spatial reliability. The change is small, and nearly imperceptible if $N<25$.

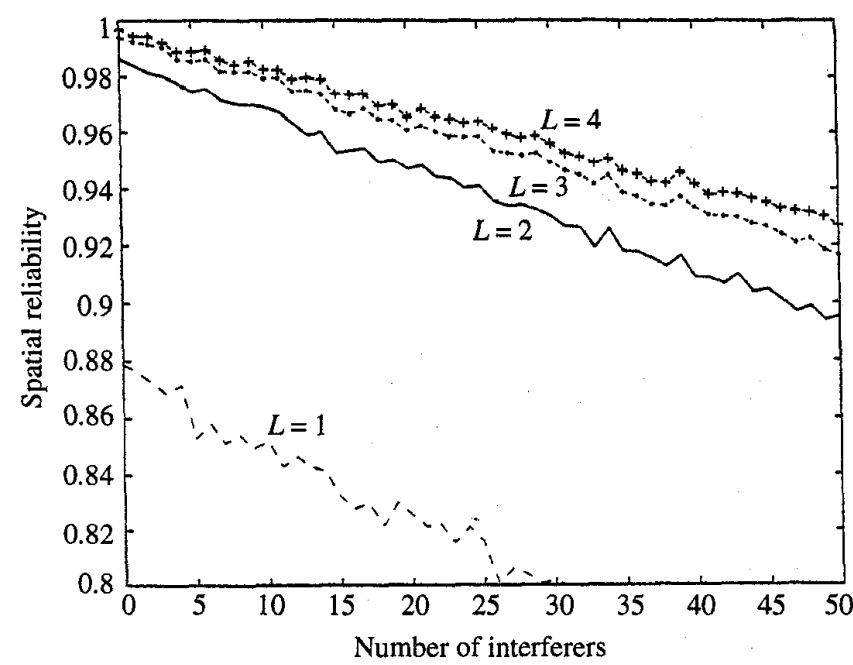

Figure 1. Spatial reliability for Rayleigh fading, $M_{1}=250$, $E=0.01$, and minimum $S N R=20 \mathrm{~dB}$.

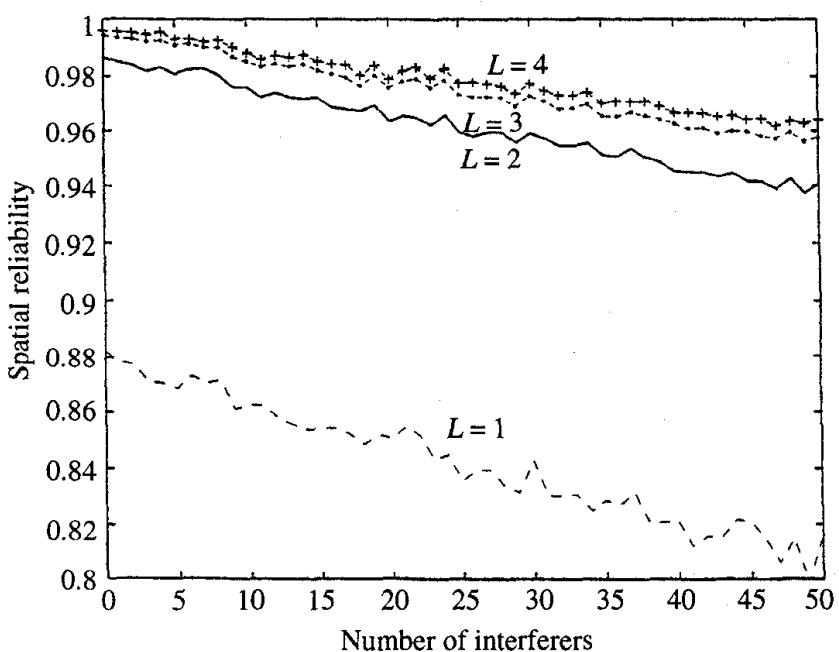

Figure 2. Spatial reliability for Rayleigh fading, $M_{1}=500$, $E=0.01$, and minimum $S N R=20 \mathrm{~dB}$. 
Figure 2 illustrates the effect of increasing the number of equivalent channels to $M_{1}=500$. Let the capacity of the network be defined as the maximum number of interfering users for which the spatial reliability is greater than or equal to 0.95 . Figures 1 and 2 and other simulation results indicate that for the parameter values selected, the capacity $C$ for dual spatial diversity is approximately proportional to $M_{1}$; specifically, $C \approx 0.07 M_{1}$ for $100 \leq M_{1} \leq 1000$.

Figure 3, which was calculated with (13), illustrates the improved performance that is possible when Rayleigh fading is absent. For Rician fading with a significant direct line-of-sight signal, we can expect a system performance intermediate between those shown in Figures 1 and 3.

Figure 4 illustrates the sensitivity of the network to an increase in the minimum SNR, which may be due to a change in $P_{0}$ or $\sigma_{n}^{2}$. For no spatial diversity or dual diversity, a substantial performance improvement occurs when the minimum $S N R=25 \mathrm{~dB}$. Other simulation results indicate that a decrease in the minimum SNR below $20 \mathrm{~dB}$ severely degrades performance.

The effect of the threshold $E$ on the spatial reliability is illustrated in Figure 5, where the threshold is doubled to $E=0.02$. The appropriate value of the threshold depends on the desired information-bit error probability and the error-correcting code.

\section{CONCLUSIONS}

A few basic conclusions about frequency-hopping CDMA communications in a mobile peer-to-peer network can be drawn. The performance is greatly improved by the use of spatial diversity, which usually requires carrier frequencies in excess of $1 \mathrm{GHz}$. A crucial parameter is the number of equivalent frequency channels, which can be increased not only by an increase in the number of frequency channels, but also by a decrease in the duty factor of the network users. The data modulation method that is most suitable appears to be MSK or some other form of CPFSK. For these modulations, $B T_{S} \approx 1$, and the scenario modeled, the spectral splatter from adjacent channels is not an important factor if the number of interferers is much smaller than the number of equivalent channels.

\section{REFERENCES}

[1] R. Wang and J.P.M.G. Linnartz, "Design of a Frequency Hopping Spread Spectrum Communication System for an Automated Highway System," Proc. Asilomar Conf., pp. 265-269, November 1996.

[2] D. Torrieri, "Future Army Mobile Multiple-Access Communications," Proc. IEEE Military Commun. Conf., pp. 650654, November 1997.

[3] J. P. Linnartz, Narrowband Land-Mobile Radio Networks. Boston: Artech House, 1993.

[4] D. Torrieri, Principles of Secure Communication Systems, 2nd ed. Boston: Artech House, 1992.

[5] F. Adachì and J. D. Parsons, "Unified Analysis of Postdetection Diversity for Binary Digital FM Radio," IEEE Trans. Veh. Technol., vol. VT-37, pp. 189-198, November 1988.

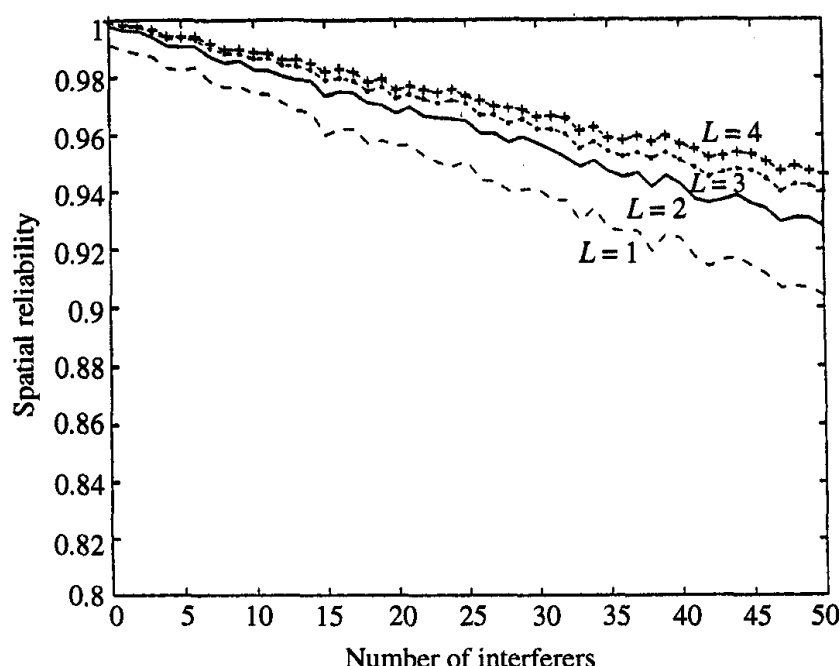

Figure 3. Spatial reliability for no fading, $M_{1}=250, E=0.01$, and minimum SNR $=20 \mathrm{~dB}$.

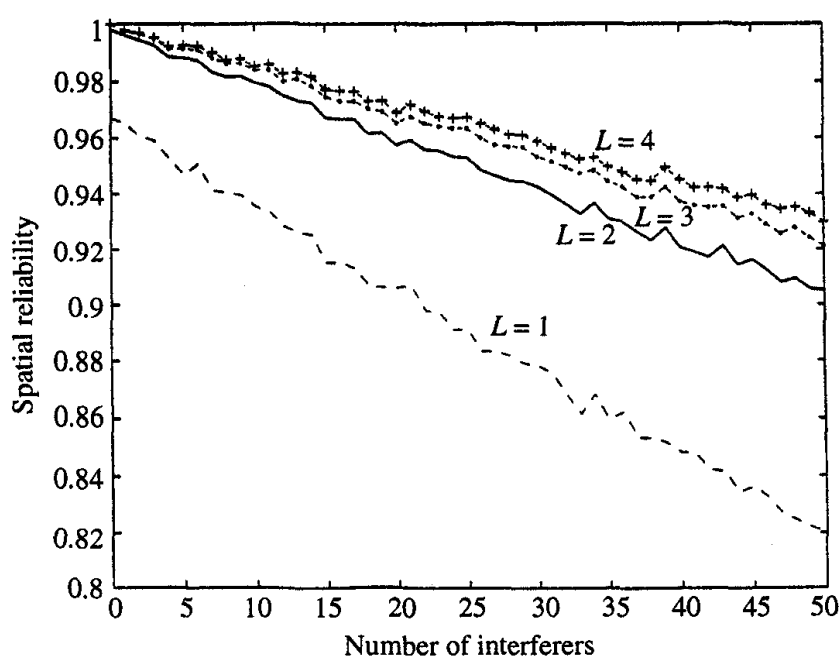

Figure 4. Spatial reliability for Rayleigh fading, $M_{1}=\mathbf{2 5 0}$, $E=0.01$, and minimum $S N R=25 \mathrm{~dB}$.

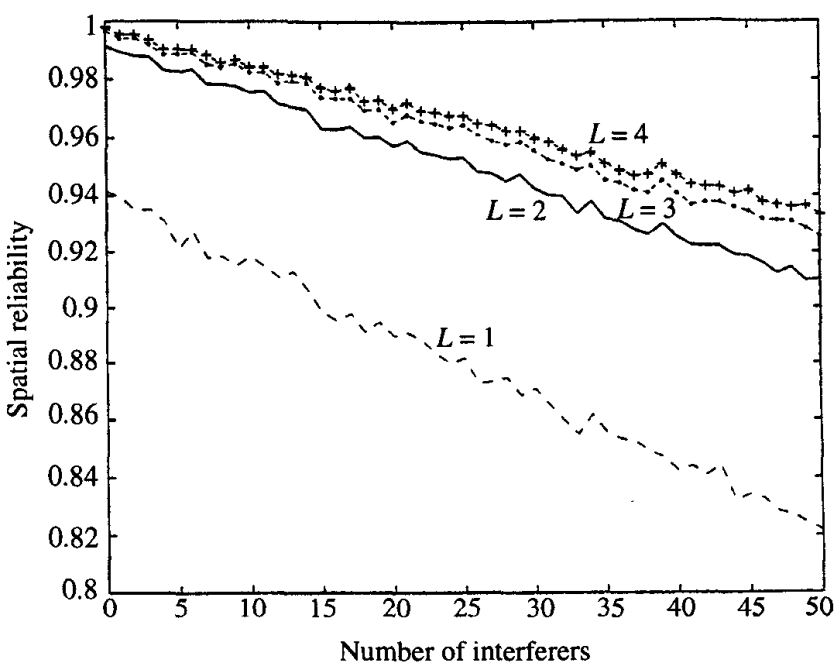

Figure 5. Spatial reliability for Rayleigh fading, $M_{1}=\mathbf{2 5 0}$, $E=0.02$, and minimum $S N R=20 \mathrm{~dB}$. 